\title{
Research Paper \\ Effect of Coenzyme Q10 Supplementation on Liver Total Oxidant/Antioxidant Status in Streptozotocin-Induced Diabetic Rats
}

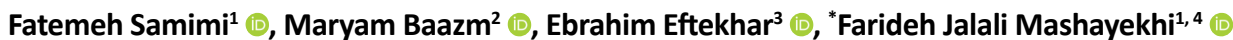

\author{
1. Department of Biochemistry and Genetics, Faculty of Medicine, Arak University of Medical Sciences, Arak, Iran. \\ 2. Department of Anatomy, Faculty of Medicine, Arak University of Medical Sciences, Arak, Iran. \\ 3. Endocrinology and Metabolism Research Center, Hormozgan University of Medical Sciences, Bandar Abbas, Iran. \\ 4. Department of Laboratory Sciences, Faculty of Paramedicine, Arak University of Medical Sciences, Arak, Iran.
}

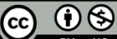

Article Info:

Received: 25 Mar 2019

Accepted: 18 Jun 2019

Available Online: 01 Oct 2019

Key words:

Coenzyme Q10, Diabetes mellitus, Total oxidant status, Total antioxidant capacity, Oxidative stress index

\section{A B STRACT}

Background and Aim Oxidative stress is the main factor in the development and progression of diabetes and its related complications. There is growing evidence that antioxidants supplementation can improve oxidative stress induced in diabetes. The present investigation was conducted to study the effects of Coenzyme Q10 (CoQ10) on the Oxidative Stress Index (OSI) in diabetic rats.

Methods and Materials A total of 30 male rats were divided into five groups: saline, sesame oil (as a vehicle), CoQ10-treated (10 mg/kg/day), diabetic (induced with streptozotocin: $55 \mathrm{mg} / \mathrm{kg}$ ), and CoQ10-treated diabetic $(10 \mathrm{mg} / \mathrm{kg} / \mathrm{d})$. Then, we measured the Malondialdehyde (MDA), Total Oxidant Status (TOS), and Total Antioxidant Capacity (TAC) levels in the rats' liver homogenate. Additionally, the OSI was calculated.

Ethical Considerations The Research Ethics Committee of Arak University of Medical Sciences approved this study (Code: IR.ARAKMU.REC.1397.119).

Results results showed a significant decrease in the level of liver MDA ( $P=0.022)$, TOS $(P=0.03)$, and OSI $(P=0.028)$ in the CoQ10-treated diabetic group compared to the diabetic rats. No significant change was observed in the total thiol group $(P=0.25)$ and TAC $(P=0.77)$ level in diabetic rats' livers treated with CoQ10 compared to diabetic rats.

Conclusion CoQ10 supplementation can reduce oxidative stress induced in the liver of diabetic rats through the suppression of TOS, OSI, and lipid peroxidation.

\section{Extended Abstract}

\section{Introduction}

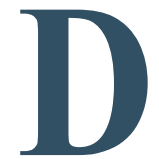

iabetes mellitus is a metabolic disease characterized by elevated blood glucose levels and impaired metabolism of carbohydrates, proteins, and lipids [1]. In diabetes, elevat- ed blood sugar increases the production of reactive oxygen species that results in oxidative stress. Research findings suggest that complications and problems of diabetes may be partly related to oxidative stress [2]. Cells contain a variety of enzymatic and non-enzymatic antioxidants. The activity of these antioxidant compounds reduces the harmful effects of free radicals and prevents their oxidative stress [3]. Coenzyme Q10 (CoQ10) is one of the essential endogenous

\section{* Corresponding Author}

Farideh Jalali Mashayekhi, PhD.

Address: Department of Biochemistry and Genetics, Faculty of Medicine, Arak University of Medical Sciences, Arak, Iran.

Tel: +98 (918) 8614706

E-mail: mashayekhi@arakmu.ac.ir 
cellular antioxidants that can neutralize all kinds of free radicals $[4,8]$. Studies indicate a close relationship between decreased CoQ10 level and the incidence of diabetes. Therefore, one of the suggested strategies to reduce the complications of oxidative stress in diabetes is to use CoQ10 [6]. The purpose of the present study was to compare the effect of CoQ10 on Total Oxidative Capacity (TOC), Total Antioxidant Status (TAS), and Oxidative Stress Index (OSI).

\section{Materials and Methods}

In this experimental study, 30 male Wistar rats were used. The rats were randomly divided into 5 groups, including three healthy control groups (saline, sesame oil [as vehicle] and CoQ10-treated [10 $\mathrm{mg} / \mathrm{kg} / \mathrm{d}$ in sesame oil]) [7], diabetic group, and CoQ10-treated diabetic group (10 mg/kg/d in sesame oil for 42 days). Induction of diabetes in rats was done by intraperitoneal injection of Streptozotocin (STZ) at a dose of $55 \mathrm{mg} / \mathrm{kg}$, and its confirmation was done by measuring blood glucose level 72 $\mathrm{h}$ and one wk after induction. The rats with fasting blood glucose levels above $250 \mathrm{mg} / \mathrm{dL}$ were considered as diabetic. At the end of the experiment, the rats were anesthetized by intraperitoneal injection of ketamine $(60 \mathrm{mg}$ / $\mathrm{kg}$ ) and xylazine (20 mg/kg). Then, their blood was taken from their hearts, and their liver tissue was also removed. Serum glucose and Malondialdehyde (MDA) levels, thiol groups, TOS, and TAC, were measured in homogenized liver tissue [14-18].

\section{Results}

Comparison of MDA levels in the study groups showed that the level of this marker significantly increased in the diabetic control group compared to the healthy control group $(\mathrm{P}=0.001)$. Treatment with $\mathrm{CoQ} 10$ in diabetic rats resulted in a significant decrease in MDA concentration compared to the diabetic control group $(\mathrm{P}=0.022)$. Moreover, the mean concentration of thiol groups in the diabetic group significantly decreased compared to control groups $(\mathrm{P}=0.001)$. Treatment with $\mathrm{CoQ} 10$ in diabetic rats had increased the concentration of thiol groups compared to the diabetic group, but this increase was not statistically significant $(\mathrm{P}=0.25)$.

The diabetic group showed a significant decrease in $\mathrm{TAC}$ compared to healthy control groups $(\mathrm{P}=0.01)$. Treatment with CoQ10 in diabetic rats increased TAC, but this increase was not statistically significant $(\mathrm{P}=0.77)$. Comparison of TOS in the study groups showed that the level of this factor in the diabetic group was significantly higher compared to that in the healthy control groups $(\mathrm{P}=0.001)$.
Treatment with CoQ10 in diabetic rats resulted in a significant decrease in TOS compared to the diabetic group $(\mathrm{P}=0.03)$. Regarding the mean OSI in study groups, the diabetic group showed a significant increase compared to healthy control groups $(\mathrm{P}=0.001)$. Treatment of diabetic rats with CoQ10 resulted in a significant decrease in OSI compared to the diabetic group $(\mathrm{P}=0.028)$.

\section{Discussion}

In diabetes, the metabolism of carbohydrates, proteins, and lipids is disrupted, and this condition eventually leads to increased oxidative stress [19]. Oxidative stress acts as a detrimental factor in exacerbating the pathological status of diabetes and liver tissue damage. CoQ10 is a fatsoluble vitamin-like compound and is found within membrane phospholipid bilayers and intracellular membranes [24]. It also prevents the oxidation of lipoproteins by reducing and regenerating vitamin E ( $\alpha$-tocopherol) [23]. Also, it can regenerate known antioxidants such as ascorbate, tocopherol, and glutathione by converting their oxidized form to reduced form. CoQ10 increases the ratio of reduced to oxidized glutathione in the liver and decreases the levels of reactive oxygen species increases the activity of mitochondrial electron transport chain complexes, and finally lowers the effects of oxidative stress [26, 27].

The results of the present study showed that the induction of diabetes in rats increased TOS level, decreased TAC level, and increased OSI value in their liver. Daily intake of $10 \mathrm{mg} / \mathrm{kg}$ CoQ10 in diabetic rats decreased TOS and MDA levels but increased TAC and decreased OSI. Therefore, CoQ10 supplementation can reduce diabetes complications by reducing lipid peroxidation and oxidative stress-induced diabetes.

\section{Ethical Considerations}

\section{Compliance with ethical guidelines}

This study obtained its ethical approval from the Research Ethics Committee of Arak University of Medical Sciences (Code: IR.ARAKMU.REC.1397.119). All ethical principles of working on laboratory animals were according to their guidelines.

\section{Funding}

This study was extracted from a research proposal (No: 3134) and was funded by the Deputy for Research and Technology of Arak University of Medical Sciences. 


\section{Authors' contributions}

All authors have met standard writing based on the guidelines of the International Committee of Medical Journal Publishers (ICMJE).

Conflicts of interest

The authors declare no conflict of interest.

Acknowledgements

The authors would like to thank the Deputy of Research and Technology of Arak University of Medical Sciences and its biochemistry laboratory experts. 


\title{
مطالعه اثر مكمل كوآنزيم Q10 بر وضعيت اكسيدانى و آنتى اكسيدانى ثام و شاخص استرس

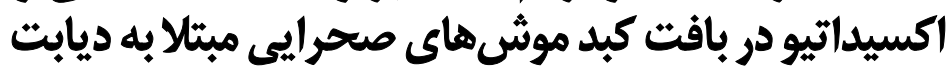

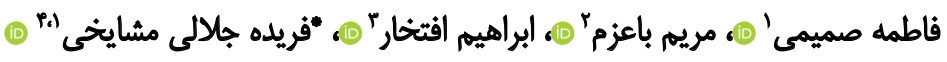

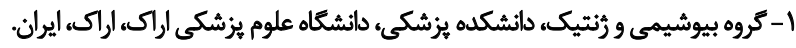

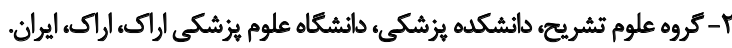

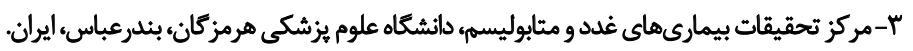

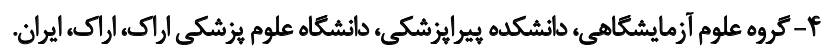

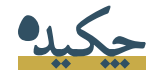

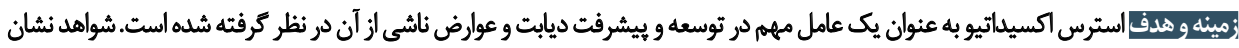

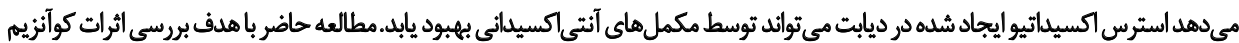

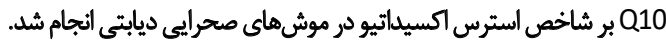

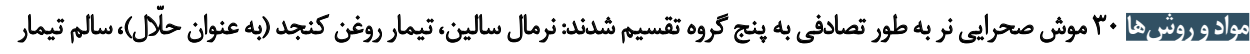

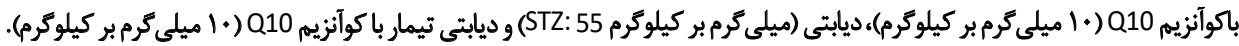

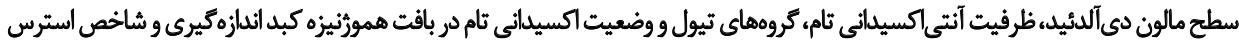
اكسيداتيومحاسبه شد.

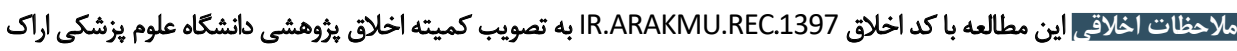
رسيدهاست.

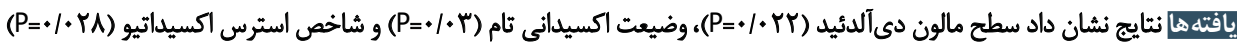

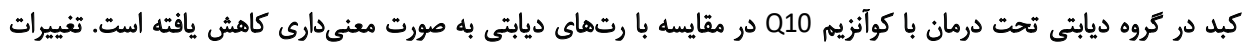

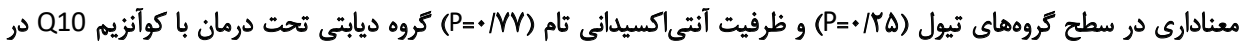

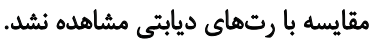

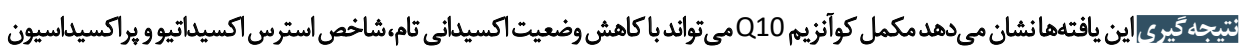

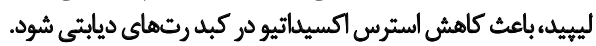

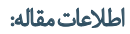
تاريخ دريافت: A • فروردين

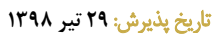

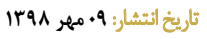

راديكال هاى آزاد اكسيرن' و بروز استرس اكسيداثيو مي دشود. نتايح

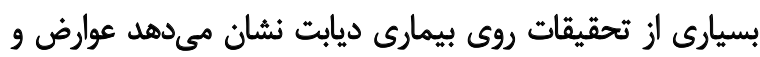

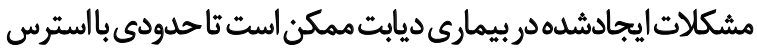

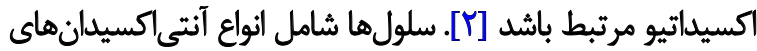

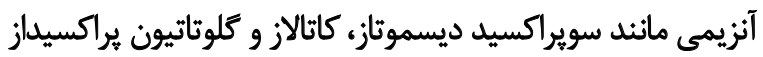

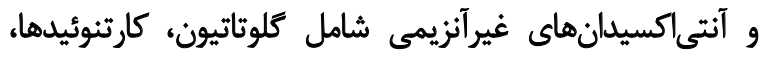

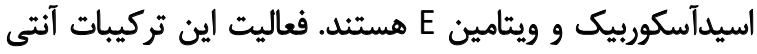

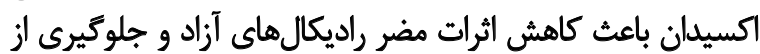

استرس اكسيداتيو مىشود [بان.

daleo

ديابت مليتوس بيمارى متابوليكى است كه ويزثكى آن

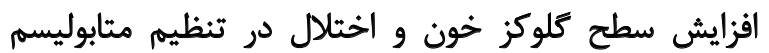

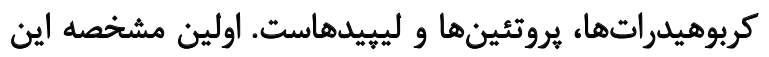

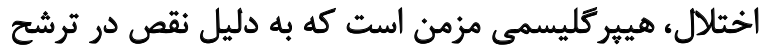

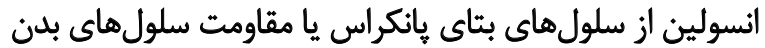

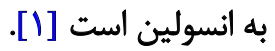
در طول ديابت افزايش مداوم قيند خون باعث افزايش توليد 
جمله كوآنزيم Q10 بر شاخص استرس اكسيداتيو در ديابت

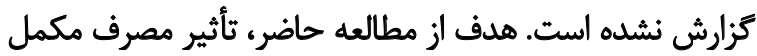

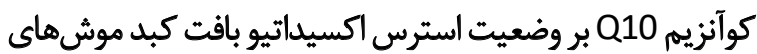

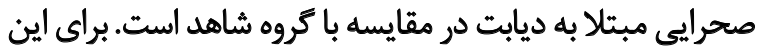

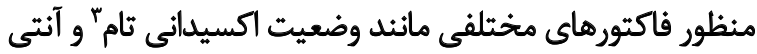

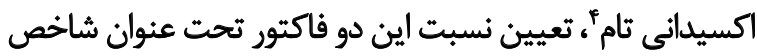

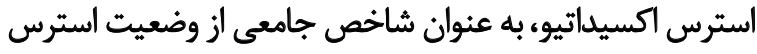

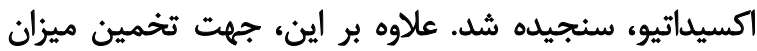

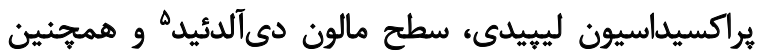

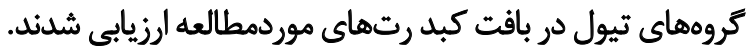

\section{مواد ورشها}

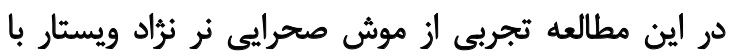

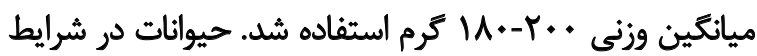

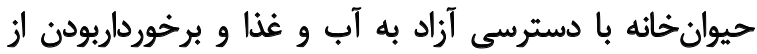

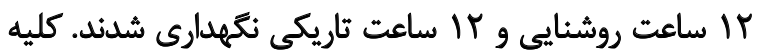

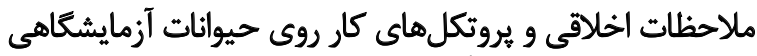

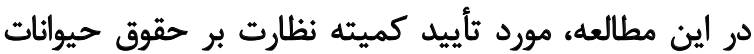

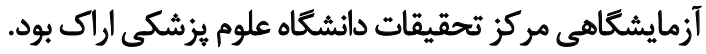

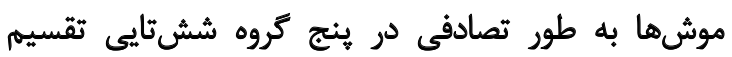

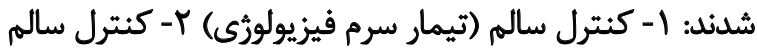

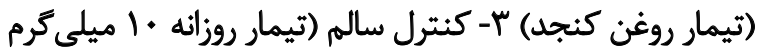

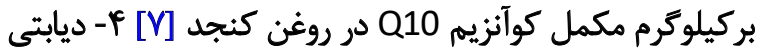

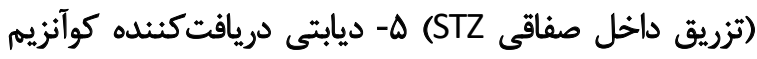

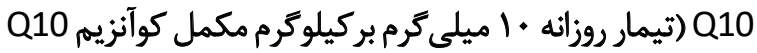

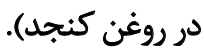

براى ديابتى كردن موشها، از محلول ثازه استريتوزوتوسين (Sigma-USA)

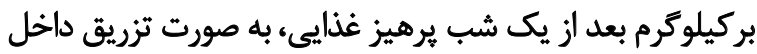

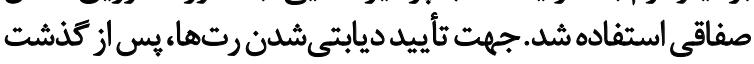

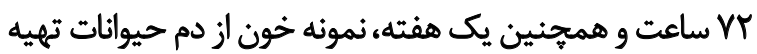

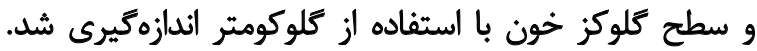

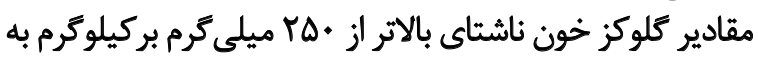
عنوان ديابت در نظر كرفته شد.

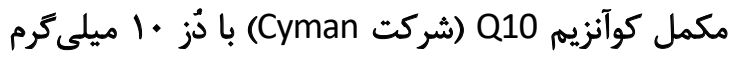

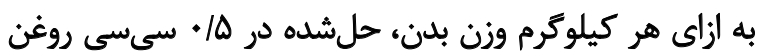

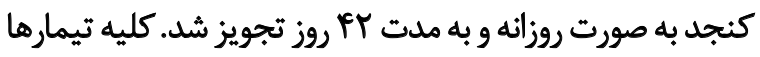

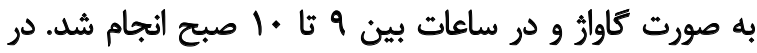

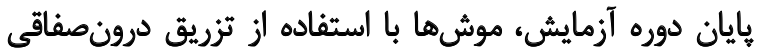

3. Total Oxidant Status (TOS)

4. Total Antioxidant Status (TAS)

5. Malondialdehyde (MDA)
در شرايط استرس اكسيداتيو، دفاع آنتىاكسيدانى سلولها،

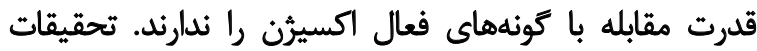

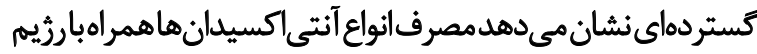

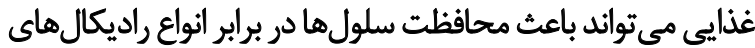

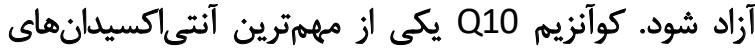

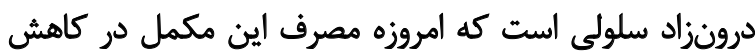

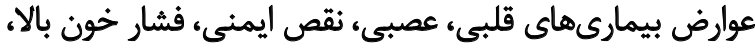

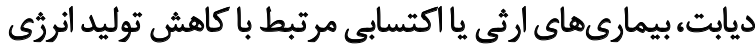

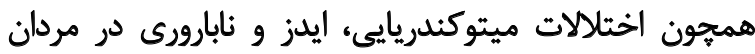

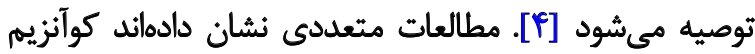

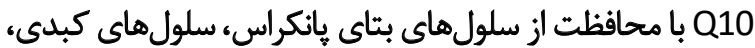

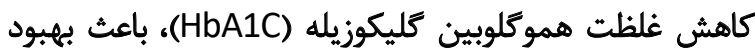

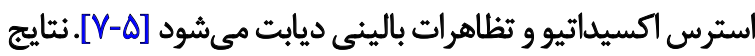

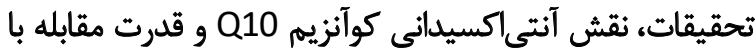
انواع راديكال هاى آزاد را تأييد مي كند [A] ماهيت آنتىاكسيداني كوآنزيم Q10 به علت مشاركت آن مولن

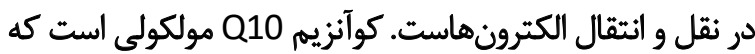

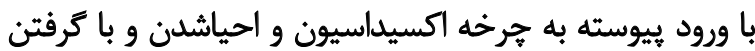

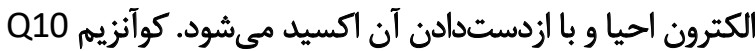

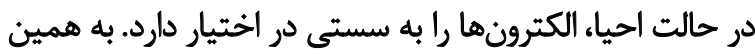

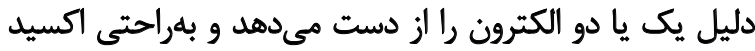

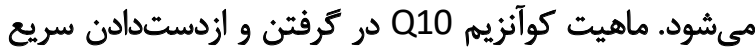

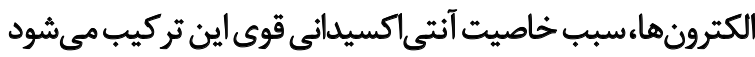

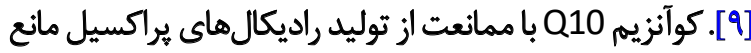

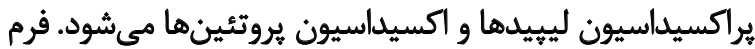

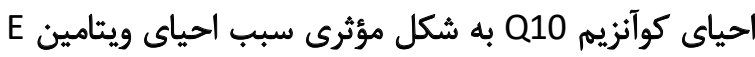

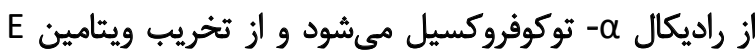

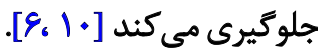

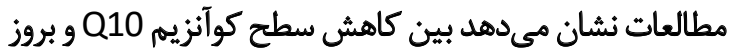

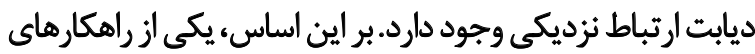

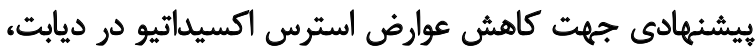

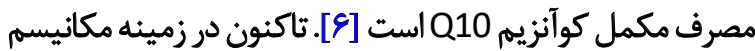

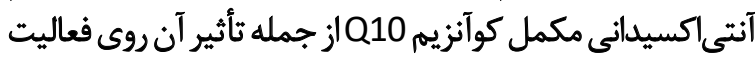

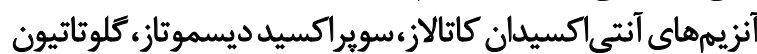

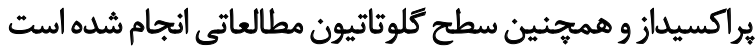

.[116. IT]

يكى از ماركرهايى كه اخيراً مورد ثوجه محققان قرار ترفته

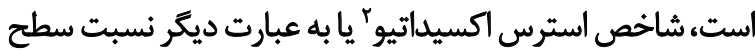

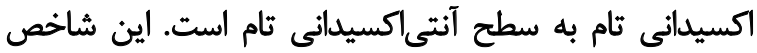

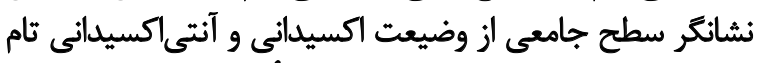

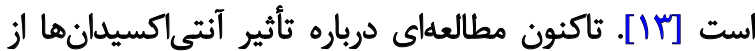

2. Oxidative Stress Index (OSI) 
ارزيابي وضعيت اكسيدانى تام به روش اسيكتروفتومترى بر هايه

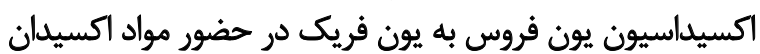

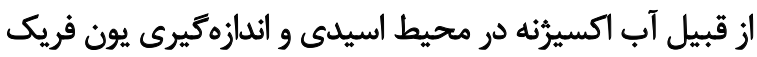

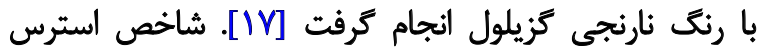

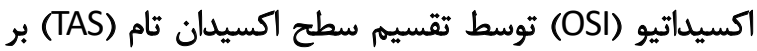

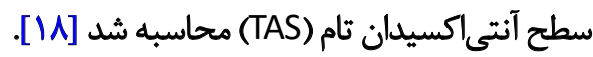

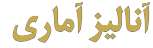

يس از جمعآورى اطلاعات و نتايج، دادهها با استفاده از نسخه

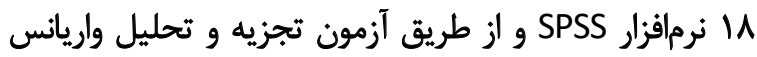

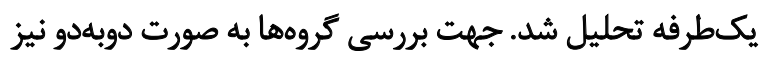

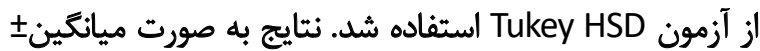

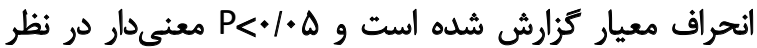

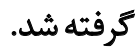

Ldịl

\section{تأثير مكمل كوآئزئم Q10 بر سطح مالون دي آلاديد بافت كبد در dellbar, go slogs}

در تصوير شماره ا ميانكين سطح متغير مالون دي ديآلدئيد

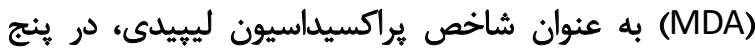
كروه تحت مطالعه نشان داده شده است. مقايسه سطح ماليد مالون

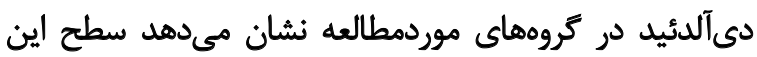

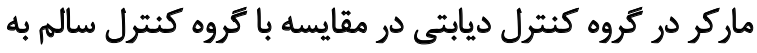

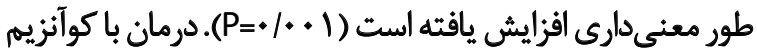

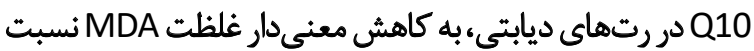

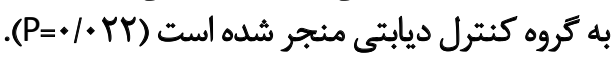

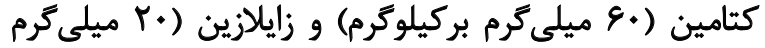

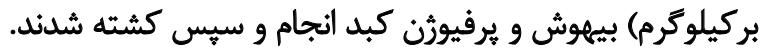

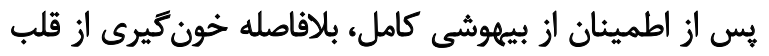

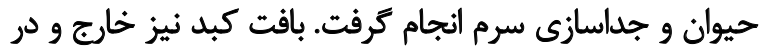

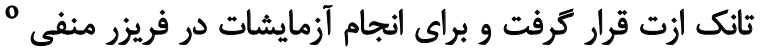

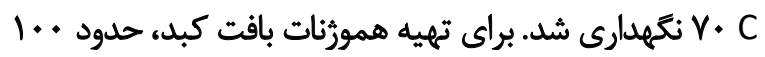

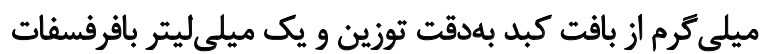

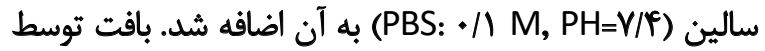

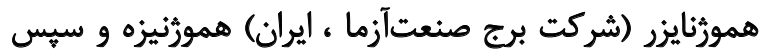

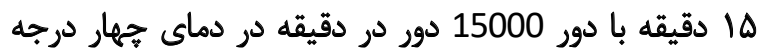

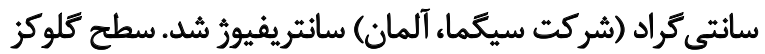

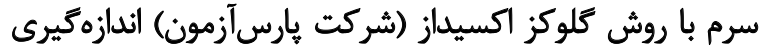

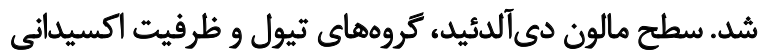

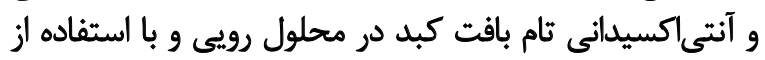
روشهاى رنكسنجى با ميكرويليت اسيكتروفتومتر (Biotek (Microplate Spectrophotometer, USA,

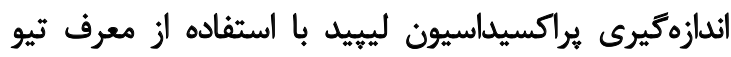

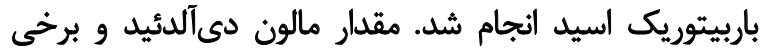

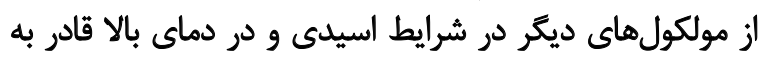

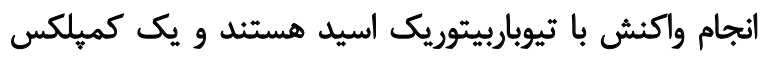

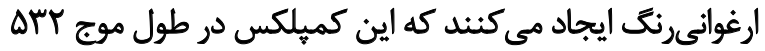

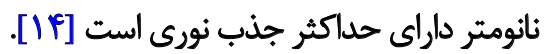

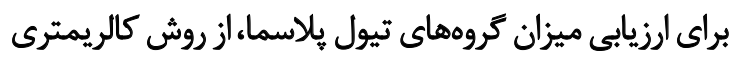
Miao-Lin Hu

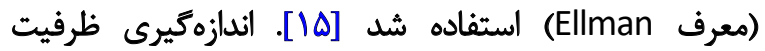

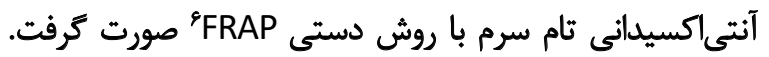

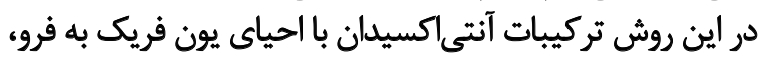

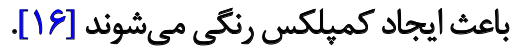

6. Ferric reducing ability of plasma

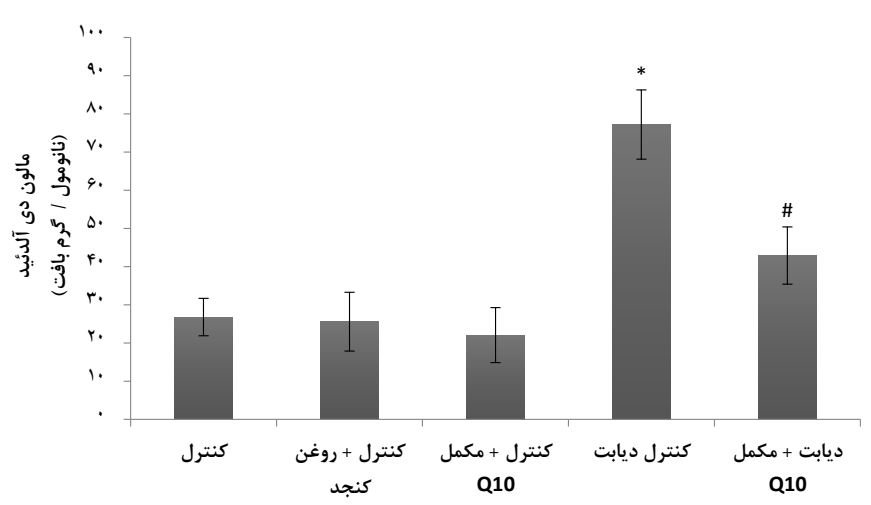

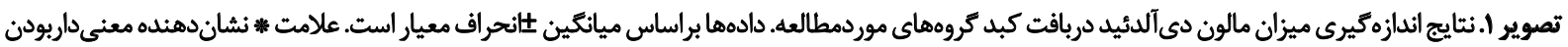

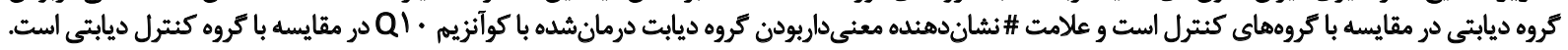




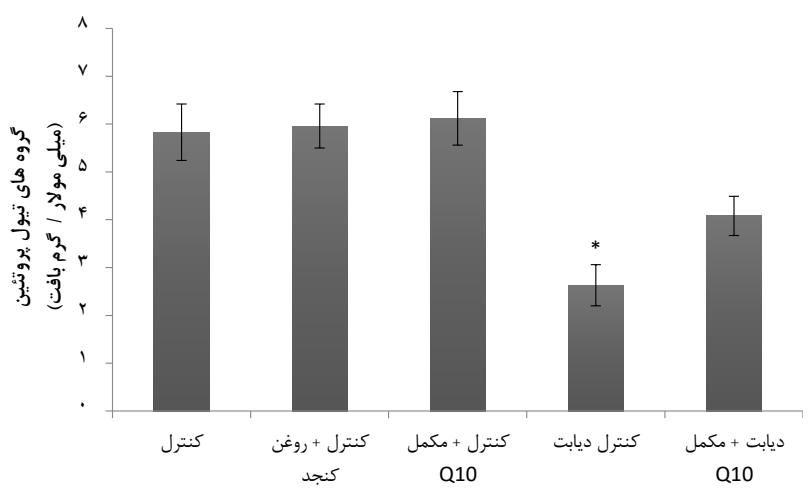

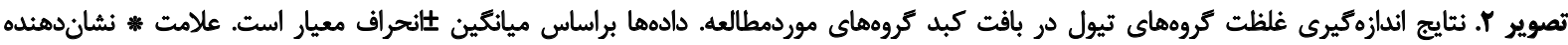

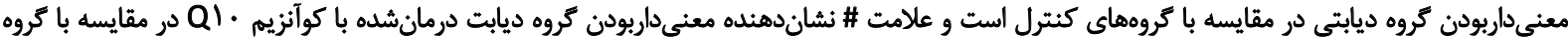
كنترل ديابتى است.

تفكيك ينج كروه تحت مطالعه نشان داده شده است. تجزيه و

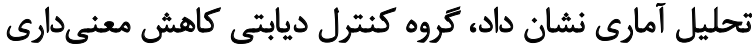

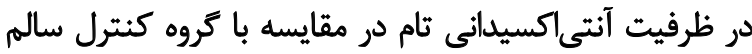

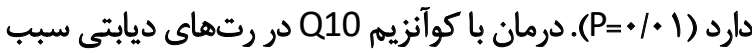

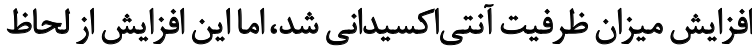

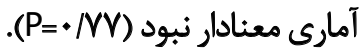

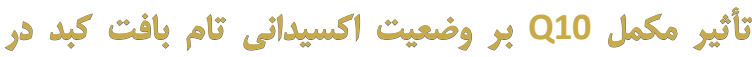

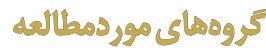

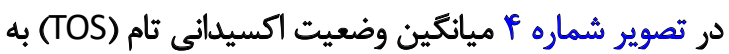

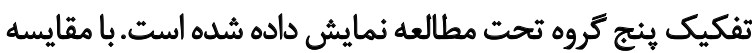

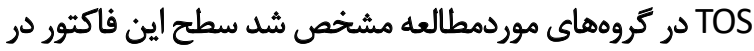

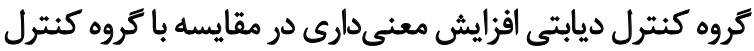

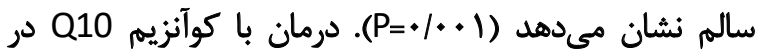

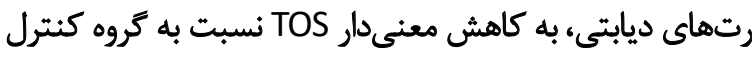

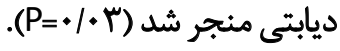

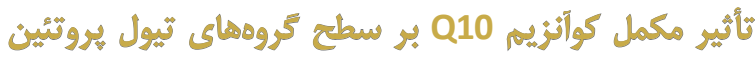

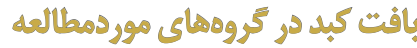

در تصوير شماره r، ميانكين سطح تروههاى تيول رادر بينج

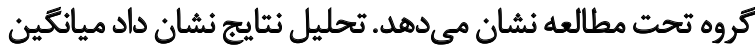

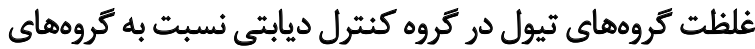

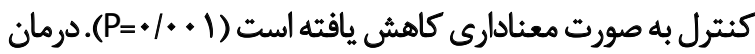

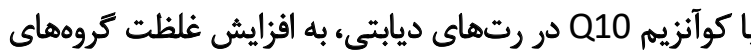

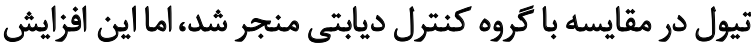

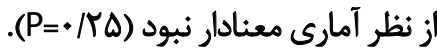

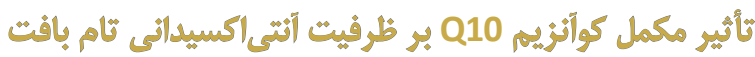

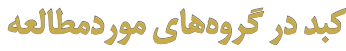

در تصوير شماره ب ميائكين ظرفيت آنتىاكسيدانى تام به

7. Total Antioxidant Capacity (TAC)

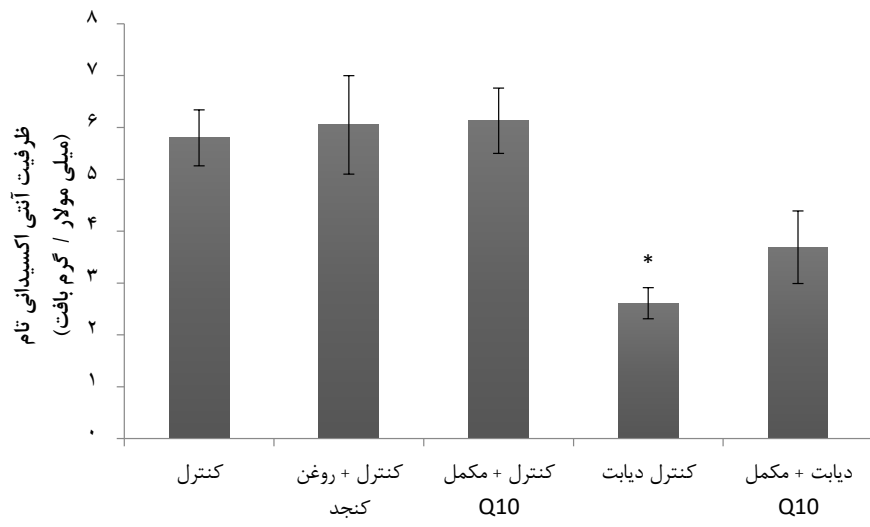

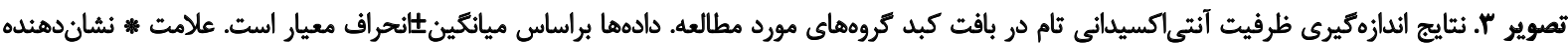

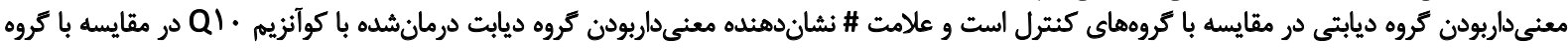
كنترل ديابتى است. 


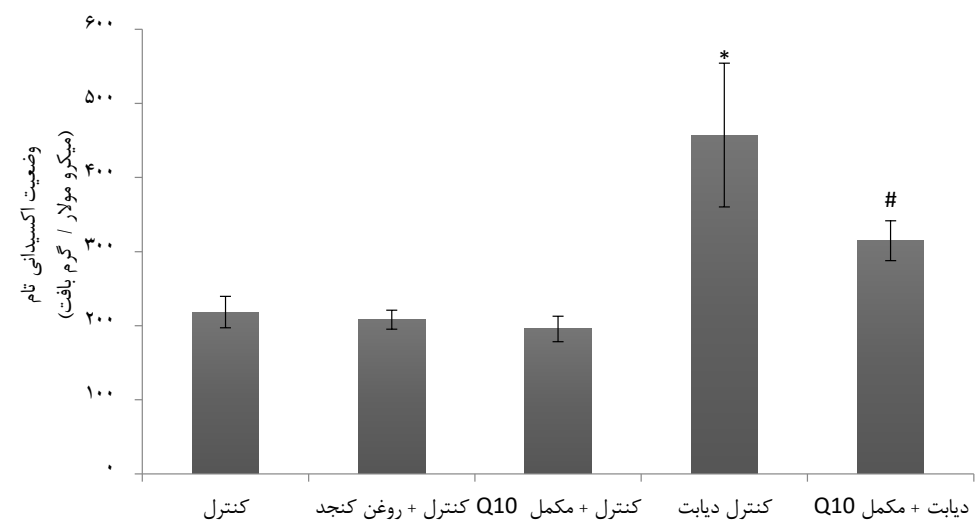

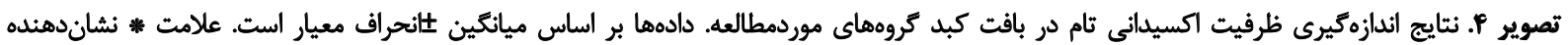

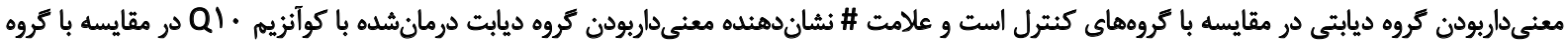

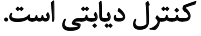

در كروه تحت درمان نسبت به گروه كنترل ديابتى مى شودو. بنابراين

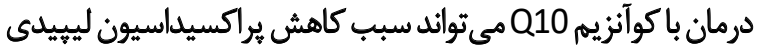

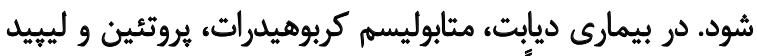

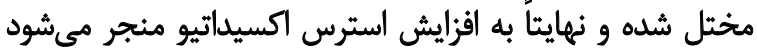

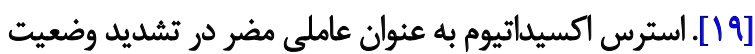

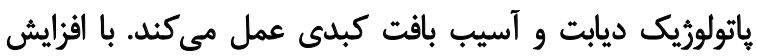

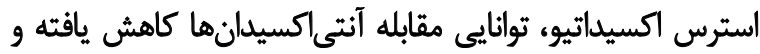

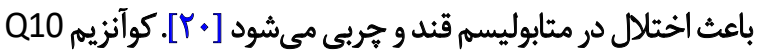

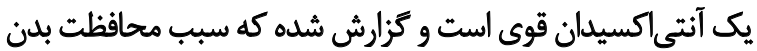

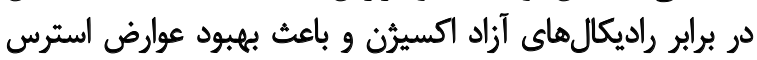

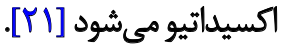

در مطالعات قبلى،بهبرخي مكانيسمهاي آنتىاكسيداني كوآنزيم

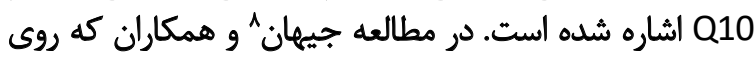

8. Jihan

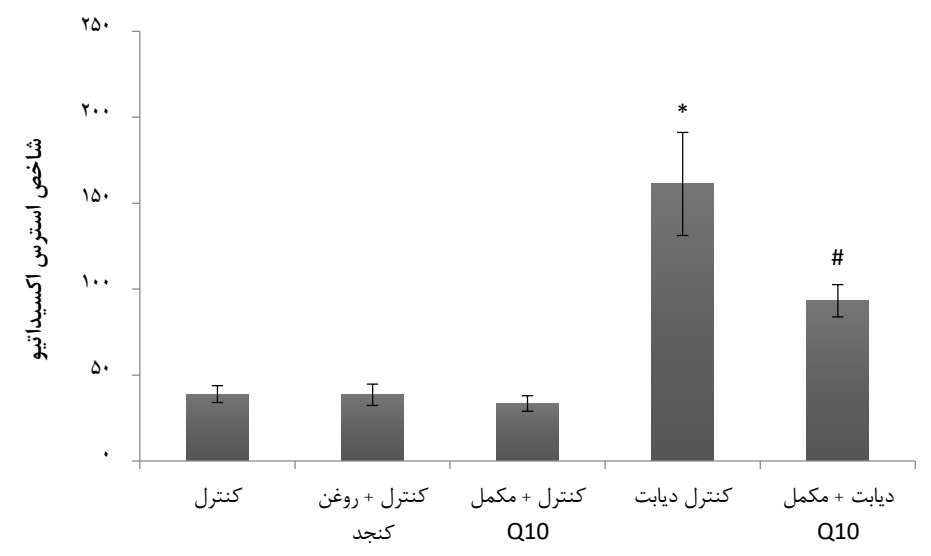

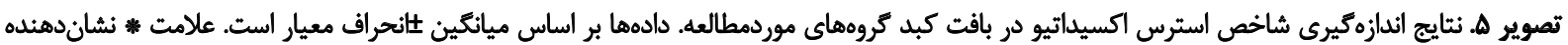

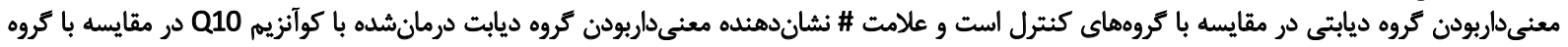
كثترل ديابتى است. 
شاخص استرس اكسيداتيو در بافت كبد رتهاي ديابتى شد. در

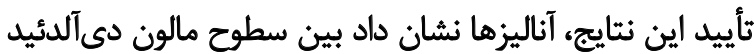

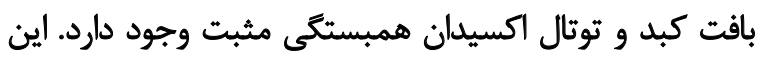

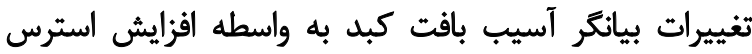

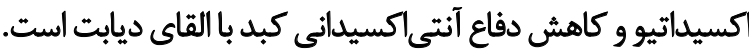

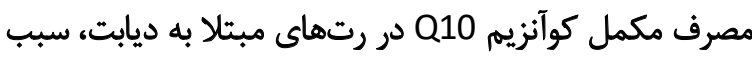

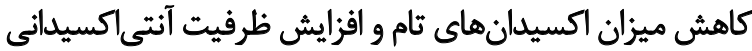

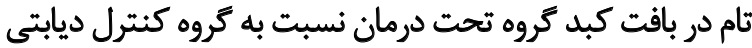

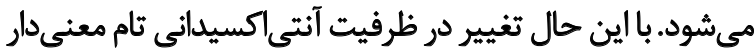

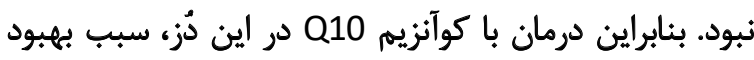
وضعيت اكسيدانى تام در رتهاي ديابتى شداني ورماء' و همكاران كاهش غيرمعنى الرار ظرفيت آنتىاكسيدانى

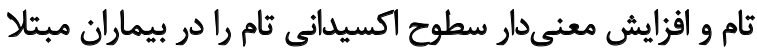

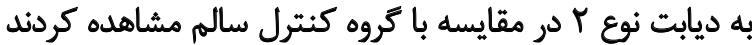

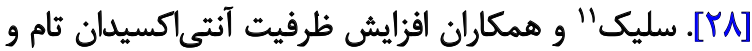

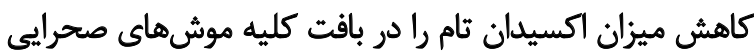

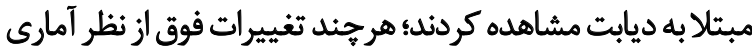

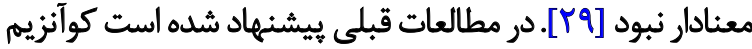

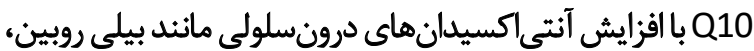

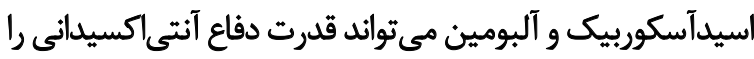

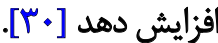
در سالهاي اخير علاوه بر مطالعه درباره ميزان آنتىاكسيدان

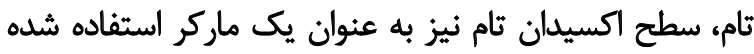

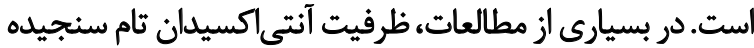

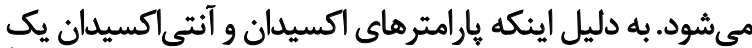

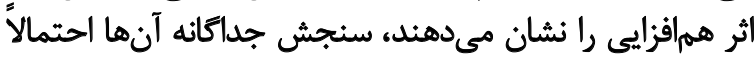

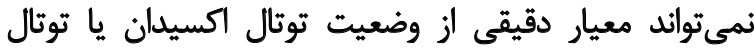

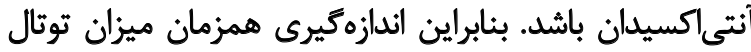

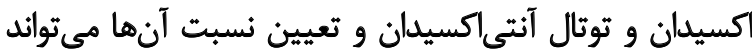

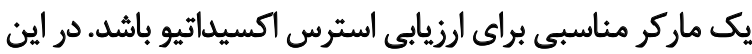

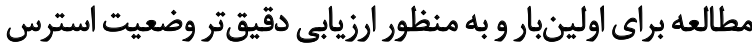

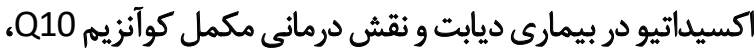
سنجش همزمان توتال اكسيدان و توتال آنتىاكسيدان و تعيين

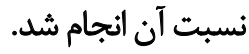

\section{نتيجليَّيرى}

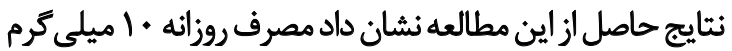

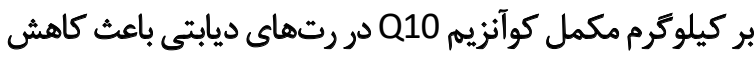

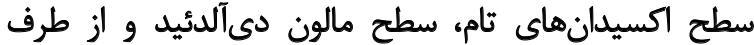
ديكر باعث افزايش ظرفيث آنتى اكسيدان تام و كاهش

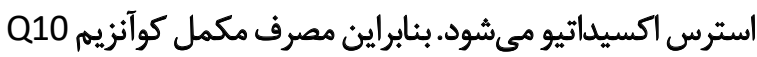

10. Verma

11. Celik
تأثير كوآنزيم Q10 بر ميزان فعاليت آنزيم سويراكسيدديسموتاز

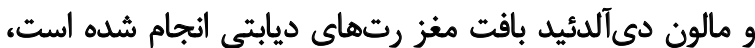

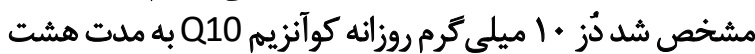

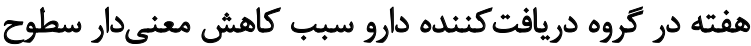

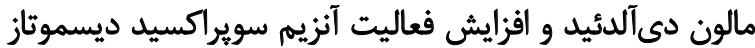

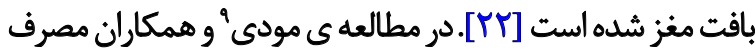

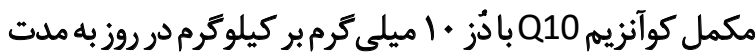

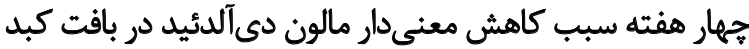

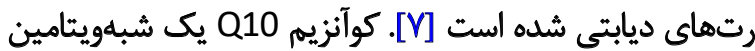

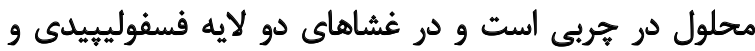

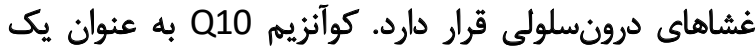

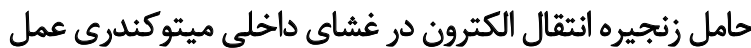

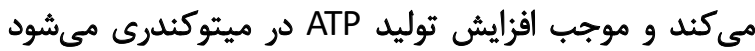

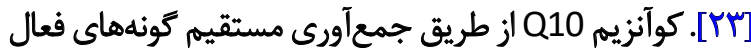

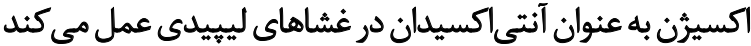

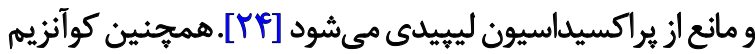

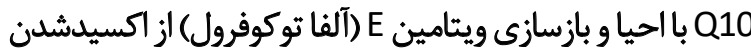
لييويروتئينها جلوكيرى مي كند [Tسازئ. نتايج اين تحقيق نشان داد مصرف مكمل كوآنزيم Q10 در دري

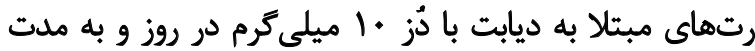

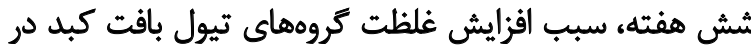
كروه تحت درمان نسبت به كروه كنته

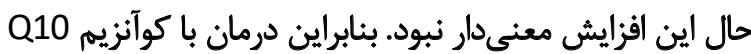

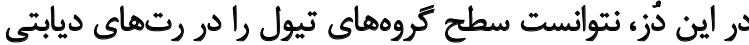

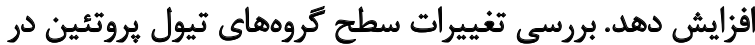

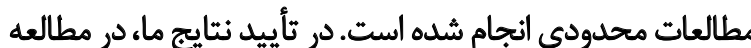

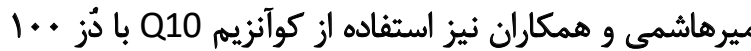

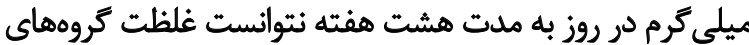

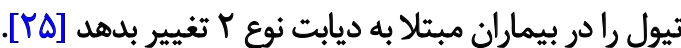

كوآنزيم Q10 يك احياكنيده قوى است و بلافاصله با باسن

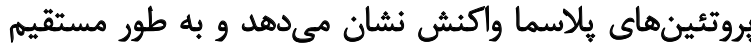

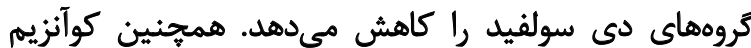

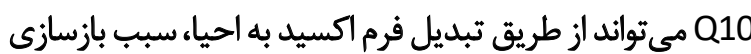

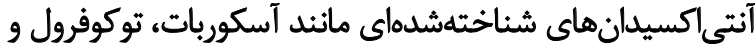

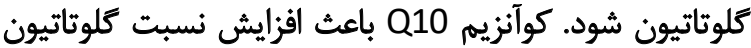

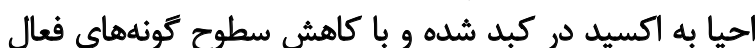

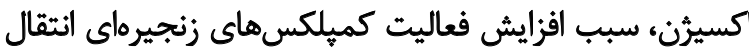

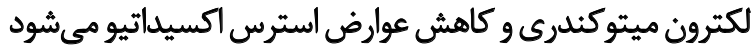

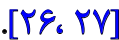

بر اساس نتايج تحقيق حاضر، القاى ديابت سبب افزايش ميزان اكسيدانهاي تام، كاهش ظرفيت آنتى اكسيداني تاني تام و افزايش 
مي تواند از طريق كاهش استرس اكسيداتيو القاشده در ديابت

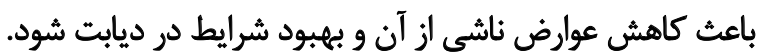

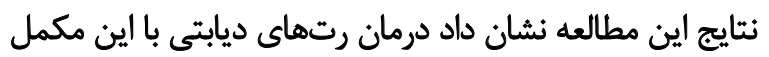

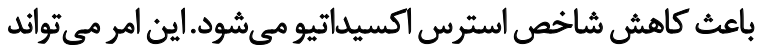

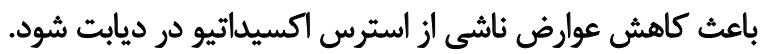

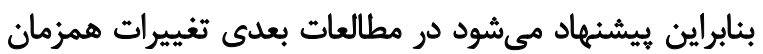

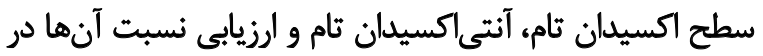

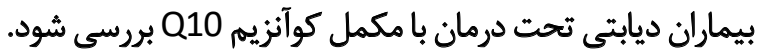

مالاحظات اخلاقى

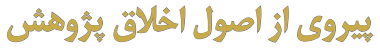

كليه ملاحظات اخلاقى و يروتكلهاى كار روى حيوانات

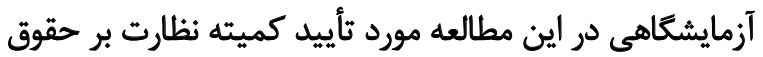

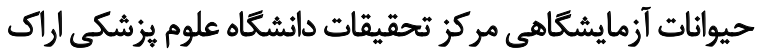

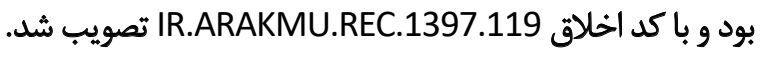

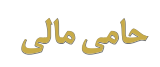

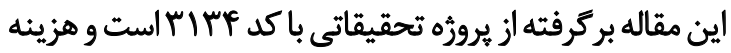

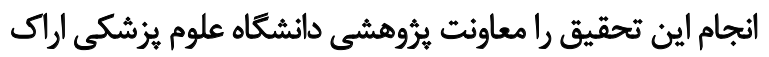
تأمين كرده است.

\section{مشاركت ثويسند مكان}

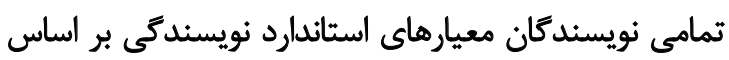

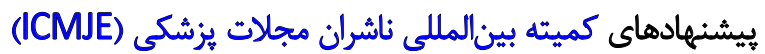
راداشتند.

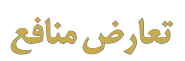

طبق نظر نويسندكان هيجگونه تضاد منافعى درباره يروهش حاضر وجود ندارد.

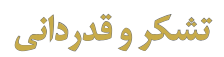
از همكارى كارشناسان آزمايشعاه بيوشيمى، سركار خانم

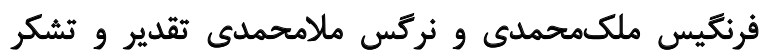
مي شود. - ميكي 


\section{References}

[1] Kerner W, Brückel J. Definition, classification and diagnosis of diabetes mellitus. Exp Clin Endocrinol Diabetes. 2014; 122(07):384-6. [DOI:10.1055/s-0034-1366278] [PMID]

[2] Pickering RJ, Rosado CJ, Sharma A, Buksh S, Tate M, de Haan JB. Recent novel approaches to limit oxidative stress and inflammation in diabetic complications. Clin Exp Immunol. 2018; 7(4):e1016. [DOI:10.1002/ cti2.1016] [PMID] [PMCID]

[3] Nimse SB, Pal D. Free radicals, natural antioxidants, and their reaction mechanisms. Rsc Advances. 2015; 5(35):27986-8006. [DOI:10.1039/ C4RA13315C]

[4] Kapoor P, Kapoor A. Coenzyme Q10-a novel molecule. J Indian Acad Clin Med. 2013; 14(1):37-45

[5] Noh Y, Kim K, Shim M, Choi S, Choi S, Ellisman M, et al. Inhibition of oxidative stress by coenzyme $Q 10$ increases mitochondrial mass and improves bioenergetic function in optic nerve head astrocytes. Cell Death Dis. 2013; 4(10):e820. [DOI:10.1038/cddis.2013.341] [PMID] [PMCID]

[6] Maedeh Moradi M, Azadbakht L. Effect of coenzyme Q10 supplementation on diabetes biomarkers: a systematic review and meta-analysis of randomized controlled clinical trials. Arch Iran Med. 2016; 19(8):588-96.

[7] Modi KP, Vishwakarma SL, Goyal RK, Bhatt PA. Beneficial effects of coenzyme Q10 in streptozotocin-induced type I diabetic rats. Iranian J Pharmacol Ther. 2006; 5(1):61-5.

[8] Sanoobar M, Eghtesadi S, Azimi A, Khalili M, Jazayeri S, Reza Gohari M. Coenzyme Q10 supplementation reduces oxidative stress and increases antioxidant enzyme activity in patients with relapsing-remitting multiple sclerosis. Int J Neurosci. 2013; 123(11):776-82. [DOI:10.3109/00207454 2013.801844] [PMID]

[9] Alam MA, Rahman MM. Mitochondrial dysfunction in obesity: potential benefit and mechanism of Co-enzyme Q10 supplementation in metabolic syndrome. J Diabetes Metab Disord. 2014; 13(1):60. [DOI:10.1186/2251-6581-13-60] [PMID] [PMCID]

[10] Nowotny K, Jung T, Höhn A, Weber D, Grune T. Advanced glycation end products and oxidative stress in type 2 diabetes mellitus. Biomolecules. 2015; 5(1):194-222. [DOI:10.3390/biom5010194] [PMID] [PMCID]

[11] Modi K, Santani D, Goyal R, Bhatt P. Effect of coenzyme Q10 on catalase activity and other antioxidant parameters in streptozotocin-induced diabetic rats. Biol Trace Elem Res. 2006; 109(1):25-33. [DOI:10.1385/ BTER:109:1:025]

[12] Coldiron Jr AD, Sanders RA, Watkins III JB. Effects of combined quercetin and coenzyme Q10 treatment on oxidative stress in normal and diabetic rats. J Biochem Mol Toxicol. 2002; 16(4):197-202. [DOI:10.1002/ jbt.10035] [PMID]

[13] Aslan M, Sabuncu T, Kocyigit A, Celik H, Selek S. Relationship between total oxidant status and severity of diabetic nephropathy in type 2 diabetic patients. Nutr Metab Cardiovasc Dis. 2007; 17(10):734-40. [DOI:10.1016/j.numecd.2006.08.005] [PMID]

[14] Jamhiri M, Hafizibarjin Z, Ghobadi M, Moradi A, Safari F. [Effect of Thymol on Serum Antioxidant Capacity of Rats Following Myocardial Hypertrophy (Persian)]. Arak Med Univ J. 2017; 20(4):10-9.

[15] Hu M, Dillard C. Plasma SH and GSH measurement. Methods Enzymol. $1994 ; 233: 385-87$

[16] Benzie IF, Strain JJ. The Ferric Reducing Ability of Plasma (FRAP) as a measure of "antioxidant power": The FRAP assay. Anal Biochem. 1996; 239(1):70-6. [DOI:10.1006/abio.1996.0292] [PMID]
[17] Heidarisasan S, Ziamajidi N, Karimi J, Abbasalipourkabir R. Effects of insulin-loaded chitosan-alginate nanoparticles on RAGE expression and oxidative stress status in the kidney tissue of rats with type 1 diabetes. IJBMS. 2018; 21(10):1035-42.

[18] Aslan R, Kutlu R, Civi S, Tasyurek E. The correlation of the Total antioxidant Status (TAS), total oxidant status (TOS) and paraoxonase activity (PON1) with smoking. Clin Biochem. 2014; 47(6):393-97. [DOI:10.1016/j. clinbiochem.2013.10.002] [PMID]

[19] Postic C, Dentin R, Girard J. Role of the liver in the control of carbohydrate and lipid homeostasis. Diabetes Metab. 2004; 30(5):398-408. [DOI:10.1016/S1262-3636(07)70133-7]

[20] Maritim A, Sanders a, Watkins lii J. Diabetes, oxidative stress, and antioxidants: A review. J Biochem Mol Toxicol. 2003; 17(1):24-38. [DOI:10.1002/jbt.10058] [PMID]

[21] Shargorodsky M, Debby O, Matas Z, Zimlichman R. Effect of long-term treatment with antioxidants (vitamin C, vitamin E, coenzyme Q10 and selenium) on arterial compliance, humoral factors and inflammatory markers in patients with multiple cardiovascular risk factors. Nutr Me tab. 2010; 7(1):1-8. [DOI:10.1186/1743-7075-7-55] [PMID] [PMCID]

[22] Hussein J, El-matty DA, El-Khayat Z, Abdel-Latif Y. Therapeutic Role of Coenzyme Q10 in Brain Injury during Experimental Diabetes. J Appl Pharm Sci. 2013; 3(6):213-7.

[23] Crane FL. Biochemical functions of coenzyme Q10. J Am Coll Nutr 2001; 20(6):591-8. [DOI:10.1080/07315724.2001.10719063] [PMID]

[24] Malm C, Svensson M, Sjöberg B, Ekblom B, Sjödin B. Supplementation with ubiquinone_10 causes cellular damage during intense exercise. Acta Physiol Scand. 1996; 157(4):511-2. [DOI:10.1046/j.1365201X.1996.534286000.x] [PMID]

[25] Mirhashemi SM, Najafi V, Raygan F, Asemi Z. The effects of coenzyme Q10 supplementation on cardiometabolic markers in overweight type 2 diabetic patients with stable myocardial infarction: A randomized, double-blind, placebo-controlled trial. ARYA Atheroscler. 2016; 12(4):158-65.

[26] Tian G, Sawashita J, Kubo H, Nishio SY, Hashimoto S, Suzuki N, et al. Ubiquinol-10 supplementation activates mitochondria functions to decelerate senescence in senescence-accelerated mice. Antioxid Redox Signal. 2014; 20(16):2606-20. [DOI:10.1089/ars.2013.5406] [PMID] [PMCID]

[27] Atmaca G. Antioxidant effects of sulfur-containing amino acids. Yonse Med J. 2004; 45:776-88. [DOI:10.3349/ymj.2004.45.5.776] [PMID]

[28] Verma AK, Chandra S, Singh RG, Singh TB, Srivastava S, Srivastava R. Serum prolidase activity and oxidative stress in diabetic nephropathy and end stage renal disease: a correlative study with glucose and creatinine. Biochem Res Int. 2014; 2014:1-7. [DOI:10.1155/2014/291458] [PMID] [PMCID]

[29] Çelik VK, Sahin ZD, Sari I, Bakir S. Comparison of oxidant/antioxidant, detoxification systems in various tissue homogenates and mitochondria of rats with diabetes induced by streptozocin. Exp Diabetes Res. 2012; 2012:1-5. [DOI:10.1155/2012/386831] [PMID] [PMCID]

[30] Sardari F, Khanevari T, Rezaei B. The Effect of Q10 Supplementation and One Bout Exhaustive Exercise on Some Oxidative (MDA) and AntiOxidative (TAC) Stress Markers in Sedentary Men. J Neyshabur Univ Med Sci. 2016; 3(4):45-55. 
This Page Intentionally Left Blank 